\title{
Memory-encoding vibrations in a disconnecting air bubble
}

\author{
Laura E. Schmidt, Nathan C. Keim, Wendy W. Zhang ${ }^{\star}$ and Sidney R. Nagel
}

\begin{abstract}
Many nonlinear processes, such as the propagation of waves over an ocean or the transmission of light pulses down an optical fibre ${ }^{1}$, are integrable in the sense that the dynamics has as many conserved quantities as there are independent variables. The result is a time evolution that retains a complete memory of the initial state. In contrast, the nonlinear dynamics near a finite-time singularity, in which physical quantities such as pressure or velocity diverge at a point in time, is believed to evolve towards a universal form, one independent of the initial state ${ }^{2}$. The break-up of a water drop in air $^{3}$ or a viscous liquid inside an immiscible oil ${ }^{4,5}$ are processes that conform to this second scenario. These opposing scenarios collide in the nonlinearity produced by the formation of a finite-time singularity that is also integrable. We demonstrate here that the result is a novel dynamics with a dual character.
\end{abstract}

We examine an experimentally accessible example: the disconnection of an air bubble from an underwater nozzle. An initial asymmetry in the neck shape excites vibrations about the symmetric dynamics. As the singularity approaches, the vibration amplitudes freeze, thus encoding information about the initial distortion. In contrast, the vibration frequencies chirp, scrambling the other half of the information. We begin by studying the disconnection dynamics without initial asymmetry. In the experiment, an air bubble is grown very slowly from a submerged nozzle until the pull of buoyancy overcomes surface tension and the bubble detaches (Fig. 1a). In this process, a column of air connecting the bubble cap and the nozzle elongates and thins before breaking apart (Fig. 1b). Figure 1c shows successive neck shapes. Initially the shape near the minimum has a generic quadratic profile. Closer to disconnection, the neck resembles two cones joined by a short cylindrical segment.

Previous studies have suggested that, when the neck is long and slender, the radial motions of water at different heights along the bubble are entirely decoupled from one another ${ }^{6,7}$. With this simplifying assumption, the time evolution of a single crosssection corresponds to the implosion of a circular void inside an inviscid liquid exterior that is subjected to an overpressure of size $\Delta p$. This is a two-dimensional (2D) version of the classical three-dimensional Rayleigh-Plesset collapse ${ }^{8}$. The void radius $R(t)$ follows a Hamiltonian evolution, with the Hamiltonian

$$
H\left(R, P_{\mathrm{R}}\right)=\left[\frac{P_{\mathrm{R}}^{2}}{2 M(R)}\right]+\Delta p \pi R^{2}+\gamma 2 \pi R
$$

where $P_{\mathrm{R}}=M(R) \mathrm{d} R / \mathrm{d} t$ is the radial momentum of the inward flow, $M(R)=2 \rho \pi R^{2} \ln \left(R_{\infty} / R\right)$ is the mass of liquid in motion, $\rho$ is the liquid density and $\gamma$ the line tension, the $2 \mathrm{D}$ analogue of surface tension. As an isolated disturbance in an Euler flow does not decay to zero at infinity, the expression for the effective mass $M(R)$ has a logarithmic dependence on $R_{\infty}$, the radial extent of the liquid exterior. This system size is taken to be much larger than all other length scales. Applying Hamilton's equations of motion $\dot{R}=\partial H / \partial P_{\mathrm{R}}, \dot{P}_{\mathrm{R}}=-\partial H / \partial R$ to $H\left(R, P_{\mathrm{R}}\right)$ yields equations governing the shape evolution. These can be recast into the form $\rho\left[\left(\dot{R}^{2} / 2\right)-\left(\dot{R}^{2}+R \ddot{R}\right) \ln \left(R_{\infty} / R\right)\right]=\Delta p+\gamma / R$, which is the $2 \mathrm{D}$ analogue of the Rayleigh-Plesset equation for spherical implosion $^{8}$. This formulation makes it clear that, in the absence of asymmetry, the implosion is integrable with one degree of freedom, the radius $R$, and one conserved quantity, the total energy $E \equiv H\left(R(t=0), P_{\mathrm{R}}(t=0)\right)$.

The initial situation most relevant for the experiment (Fig. 1) corresponds to a large void inside a quiescent liquid exterior. The potential energy term due to the overpressure $\Delta p$ dominates in the first instants. As the implosion proceeds and $R$ decreases, the line-tension term becomes negligible. Eventually, $R$ becomes so small that only the kinetic energy (the term in square brackets) dominates and equation (1) simplifies to

$$
\frac{P_{\mathrm{R}}^{2}}{2 M(R)}=\frac{M(R)}{2}\left(\frac{\mathrm{d} R}{\mathrm{~d} t}\right)^{2}=E
$$

The implosion process described by equation (1) converts the initial potential energy due to $\Delta p$ into kinetic energy during the final closure. Equation (2) also shows clearly that closure corresponds to the formation of a finite-time singularity in the mathematical model. The effective mass $M(R)$ decreases to zero as $R$ goes to zero. As a result, the closure rate $\mathrm{d} R / \mathrm{d} t$ diverges to ensure energy conservation.

In Fig. 1c, we compare the shape evolution predicted by the model, equation (2), with the experiment (see the Methods section). The calculated profiles are in good agreement with the experiment, except for a slight up-down asymmetry, probably owing to the 2D model ignoring the effect of the hydrostatic pressure gradient. This demonstrates that the measured disconnection essentially corresponds to that predicted by an idealized model that identifies the disconnection with an integrable singularity.

We next show that a perturbation from circular symmetry to the $2 \mathrm{D}$ implosion yields vibrations that change the character of the disconnection (see the Methods section). A shape slightly distorted from a perfect circle is represented as a sum of Fourier modes, $r=S(\theta, t)=\bar{R}(t)+\sum_{n} a_{n}(t) \cos \left(\phi_{n}(t)\right) \cos (n \theta)$, where $\bar{R}(t)$ is the average radius, $a_{n}(t)$ is the amplitude and $\phi_{n}(t)$ is the phase of the $n$th Fourier mode (Fig. 2a). When the distortion is small, the averaged implosion dynamics is the same as that for a circular void, so that $\bar{R}(t)$ is also described by equations (1) and (2). Also, because the interface is on average accelerating inwards radially, the different Fourier modes have the form of standing waves. A distorted void vibrates as it implodes. This vibration is induced 

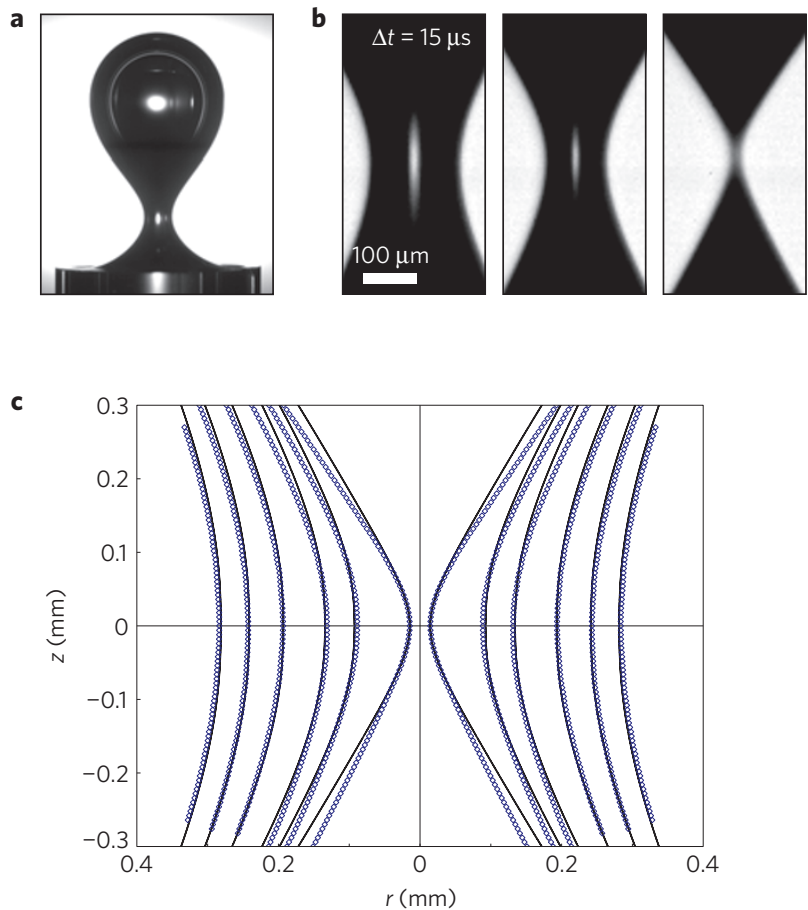

Figure 1 | Cylindrically symmetric disconnection dynamics. a, Image of an air bubble detaching from a submerged circular nozzle. The bubble is lit from the back, so the bright spots are optical artifacts. b. Close-up images of the bubble neck at different times before disconnection, separated by $15 \mu \mathrm{s}$. c, Measured profiles (diamonds) of the bubble neck at 240, 180, 120, 60,30 and $1 \mu$ s (outer to inner) before disconnection. The solid lines are profiles calculated using equation (2).

and sustained by the disturbance flow and the azimuthal variations in the exterior pressure field. Because both effects are inertial in origin, the vibrations found here are fundamentally different from the more familiar, surface-tension-induced shape vibrations first studied by Rayleigh ${ }^{9}$. In our case, the only effect of surface tension is to modify slightly the transient dynamics connecting the initial state to the final singularity.

As information about the initial state is encoded by the amplitudes and the phases of the Fourier modes, their time evolution reveals how such information survives near a singularity. First consider the amplitudes. We find that, as $t$ approaches $t_{*}$, the moment when a circular void shrinks to a point, the amplitudes of all the modes 'freeze',

$$
\lim _{t \rightarrow t_{*}} \frac{\mathrm{d} a_{n}}{\mathrm{~d} t} \rightarrow 0
$$

As a result of this saturation, the weakly perturbed void closure dynamics has a countably infinite set of constants of motion. Together they encode half of the information about the initial distortion from circular symmetry. This freezing also causes the void to become more distorted as $\bar{R}$ goes to zero. In Fig. $2 \mathrm{~b}$ we plot void shapes at successive times after they have been rescaled by $\bar{R}$. Initially the void is nearly a circle. As time goes on, the void vibrates and evolves into a self-contact, in which opposing sides of the void surface touch in a finite amount of time. The cylindrically symmetric singularity at $t_{*}$ is pre-empted. The presence of an asymmetry, however small, is therefore predicted to change the nature of the singularity entirely.

Next consider the phases of the different Fourier modes. As the singularity approaches, the frequency of the $n$th vibrational mode 'chirps',
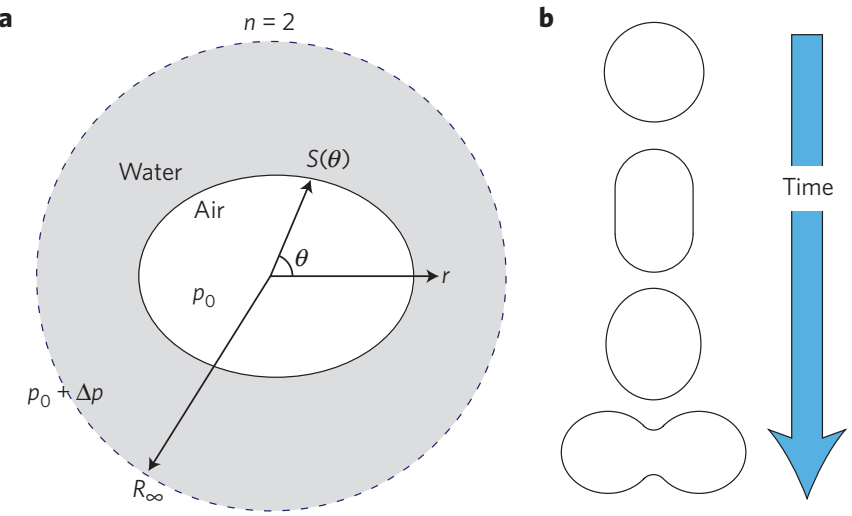

Figure $\mathbf{2}$ | Linear stability analysis of perturbed implosion. a, The void shape, $S(\theta)$, is perturbed from a circle by an $n=2$ Fourier mode (black solid line). b, Void shapes at successive times, rescaled by the average radius. As time goes on, the void vibrates and evolves into side-by-side lobes.

$$
\lim _{t \rightarrow t_{*}} \frac{\mathrm{d} \phi_{n}}{\mathrm{~d} t} \rightarrow \frac{\sqrt{n-1}}{\bar{R}(t)} \frac{\mathrm{d} \bar{R}}{\mathrm{~d} t}
$$

The frequency increases over time, becoming infinite as the singularity approaches. Equivalently, the phase $\phi_{n}(t)=\sqrt{n-1} \ln [\bar{R}(t) /$ $\bar{R}(t=0)]$. As a consequence, backing out the relation between the different phases in the initial distortion requires higher and higher precision in the values of $\phi_{n}(t)$ as $\bar{R}$ goes to zero. In this sense, half of the information about the initial state, the half encoded in the initial phase relations, is lost.

Both the freezing of the amplitudes and the chirping of the frequencies are also found in the experiment. We choose an experimental geometry that corresponds to the perturbation of a circular implosion by an $n=2$ mode. This is accomplished by growing an air bubble slowly from a slot-shaped nozzle (Fig. 3a). Because the bubble is attached to a slot, the cross-section of the neck in the neighbourhood of the minimum is an oval (Fig. 3b), instead of a circle (as was the case in the experiment shown in Fig. 1). We track the neck shape by using two high-speed cameras positioned at right angles to each other.

Figure 4a shows two sets of data-the average radius at the minimum, $\bar{R}$, for a bubble released from a slot (Fig. 3), compared with one released from a circular nozzle (Fig. 1). The two data sets show the same behaviour on a log-log plot, indicating that the average dynamics produced by a slot release is indistinguishable from the cylindrically symmetric, integrable case. They are also consistent with results from other studies on circular nozzles ${ }^{10-15}$. Figure $4 \mathrm{~b}$ plots $\Delta R$, the difference in the neck radii obtained from the two orthogonal views, versus the average radius $\bar{R}$. Initially, surface tension and buoyancy effects are significant and $\Delta R$ decreases rapidly over time, showing that the neck is evolving towards a circular crosssection. Later, as inertial effects associated with the disconnection become significant, $\Delta R$ no longer decreases monotonically but oscillates about zero with an approximately constant amplitude. Although the amplitude of this shape vibration is only a few micrometres, it is highly reproducible. A total of 42 measurements trace out the same curve. The saturation of the oscillation amplitude in $\Delta R$ is consistent with the freezing of the vibration amplitude (equation (3)). The oscillation period seems constant over $\ln (\bar{R})$, consistent with the chirping of the vibration frequency (equation (4)). Finally, we quantitatively compare the measured vibration against the linear stability results (see the Methods section). The calculated curve, the solid line in Fig. $4 \mathrm{~b}$, is in excellent agreement with the experiment. 


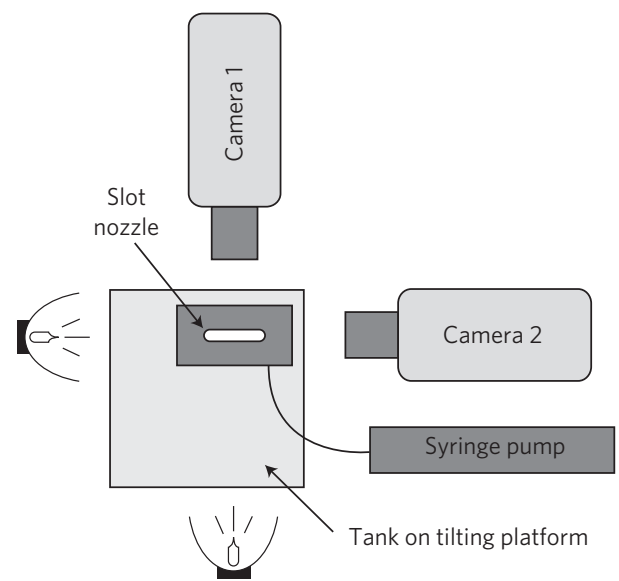

b

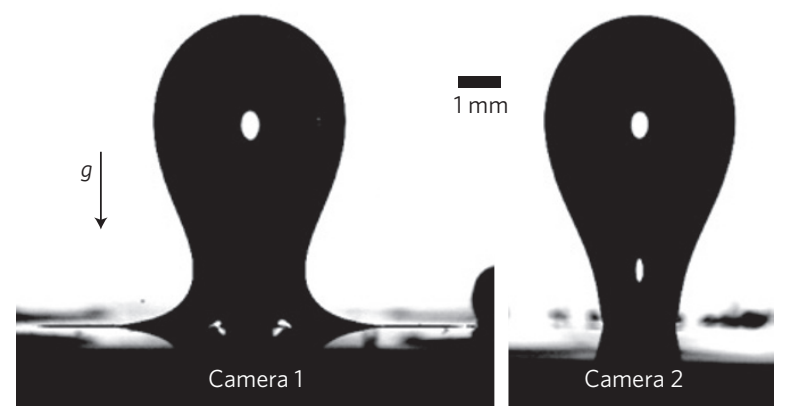

Figure 3 | Set-up of experiment used to measure an $\boldsymbol{n}=\mathbf{2}$ vibration.

a, Schematic diagram: a bubble is blown slowly from a slot-shaped nozzle, back-lit and imaged with two high-speed cameras. $\mathbf{b}$, Images of bubble while it is attached to this slot nozzle. The cross-section of the neck is extended along the slot.

The unusual vibration modes found here are natural consequences of nearly integrable Hamiltonian dynamics. Previous analyses have shown that, when vorticity effects are absent, the evolution of an air-water interface is Hamiltonian ${ }^{16}$. This means that the time evolution of the void surface when the surface is slightly distorted from a perfect circle corresponds to a nearly integrable Hamiltonian dynamics. According to the KolmogorovArnold-Moser theorem, the complete memory possessed by an integrable Hamiltonian evolution is not destroyed by a small perturbation to the Hamiltonian, regardless of the specific form of the perturbation ${ }^{17}$. Instead, the memory is largely preserved. Specifically, almost all of the trajectories in the phase space, which describe the time evolution of the system for various initial conditions, survive under very general conditions on the form of the perturbation. Only a special set of trajectories, which are evenly distributed throughout phase space and correspond to 'resonant tori', are destroyed. Typically, this persistence of memory is evident only in terms of transformed variables. What is gratifying about bubble disconnection is that, because it is associated with a finite-time singularity, the preservation of memory is apparent in a straightforward physical effect, the freezing of the vibration amplitudes.

As the most efficient way to concentrate energy is to work with a dynamics with high spatial symmetry, many energy-focusing hydrodynamic singularities are integrable. They therefore should also support memory-encoding vibrations. We have checked this by looking at the shape-stability spectrum of three previously analysed examples: the collapse of a spherical bubble ${ }^{8}$ and the implosion of cylindrical ${ }^{18}$ and spherical shock waves ${ }^{19}$. In every case, the relative

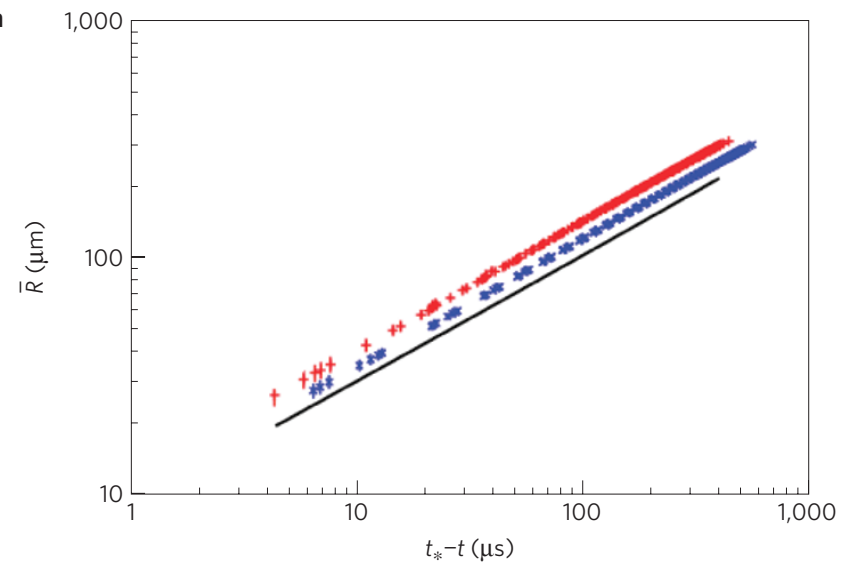

b

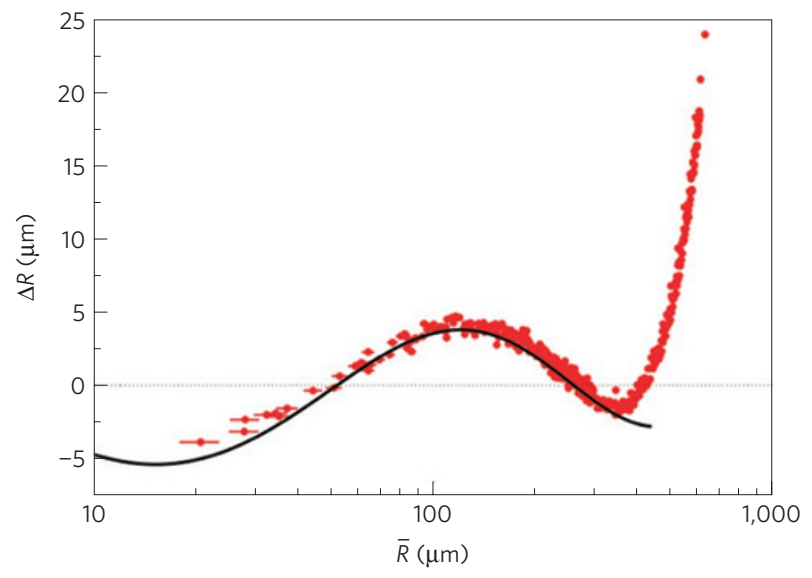

Figure 4 | Disconnection dynamics from a slot nozzle. a, Average radius at the minimum of the bubble neck produced by disconnection from a slot nozzle (red plus signs) and radius at the minimum of the cylindrically symmetric neck produced by disconnection from a circular nozzle (blue crosses) as a function of time before disconnection $t_{*}-t$. The solid line is the calculation using equation (2) with $E=13$ dynes (curve displaced downwards for clarity). b. The measured difference between the neck radius from the two orthogonal views, $\Delta R$, as a function of the averaged radius $\bar{R}$ (14 different data sets are included). The solid black line shows the linear stability result.

amplitudes of the vibrations freeze whereas the frequencies chirp as the singularity approaches. (In the collapse of a spherical bubble and a spherical shock wave the absolute scale of the mode amplitudes increases weakly as the singularity approaches. This does not affect the memory-encoding mechanism, because the relative amplitudes of the vibrational modes are preserved.)

These results suggest that memory-encoding vibrations should be a generic feature of energy focusing and may provide a rubric for thinking about the role of asymmetry in more complex processes, such as the development of a supernova ${ }^{20-22}$, jetting due to the collapse of a cavity in granular matter ${ }^{23,24}$ and inertial fusion implosion ${ }^{25}$.

In contrast to the situations listed above, the bubble disconnection process analysed here is far more tractable for experimental investigation, making it possible for us to excite and measure one of the memory-encoding vibrational modes directly. This example reveals that, under competing demands of complete memory in integrable systems and the universal dynamics near a singularity, nature reaches a Solomonic decision. Half of the information about the early history is preserved by the vibrational mode amplitudes, whereas the other half, corresponding to the phases of the different vibrational modes, is scrambled. 


\section{Methods}

Linear stability analysis. The shape of a distorted void can be written as $r=S(\theta, t)=\bar{R}(t)+\sum_{n=2}^{\infty} b_{n}(t) \cos (n \theta)$, where $\bar{R}(t)$ is the average radius and the coefficients $b_{n}(t) \equiv a_{n}(t) \cos \left(\phi_{n}(t)\right)$. As the exterior flow is incompressible and irrotational, the velocity field $\mathbf{U}=\nabla \Phi$, where the velocity potential $\Phi$ satisfies Laplace's equation $\nabla^{2} \Phi=0$. The velocity potential can be written as a sum of fundamental modes $\Phi(r, \theta, t)=Q(t) \ln (r)+\sum_{n=2}^{\infty} c_{n}(t)\left[\cos (n \theta) / r^{n}\right]$, where $Q(t)$ is the volumetric flow-rate and the coefficients $c_{n}(t)$ are related to azimuthal variations in the velocity field. The kinematic boundary condition, $\dot{S}=\left.\mathbf{U} \cdot \mathbf{n}\right|_{r=S(\theta, t)}$ where $\mathbf{n}$ is the surface normal and the overdot indicates differentiation with respect to time, yields $Q(t)=\bar{R} \bar{R}$ and $c_{n}(t)=-\bar{R}^{n+1}\left[b_{n}+b_{n}(\bar{R} / \bar{R})\right] / n$. The evolution equation for $b_{n}(t)$ follows from the normal-stress condition on the interface. For an interface in 2D with line tension $\gamma$, the exterior pressure $p(r, t)$ at the interface equals the void pressure $p_{0}$ plus a Laplace pressure contribution $\gamma \kappa$, where $\kappa$ is the 2D line curvature. To impose this pressure boundary condition, we first rewrite the momentum equation in terms of $\Phi$ and the exterior pressure $p$. This enables us to integrate the equation once over the spatial variables. The resultant Bernoulli integral relates $p$ to derivatives of $\Phi$ as follows: $\left.\rho\left[\partial \Phi / \partial t+|\nabla \Phi|^{2} / 2\right]\right|_{r=S(\theta, t)} ^{r=R_{\infty}}=-\left[p\left(r=R_{\infty}\right)-p(r=S(\theta, t))\right]$. The right-hand side of the equation is the difference in pressure from the far field, idealized as a surface at $r=R_{\infty}$ with $p=p_{0}+\Delta p$, to the void surface where $p=p_{0}-\gamma \kappa$. When the void is nearly circular $\left(\left|b_{n}(t) / \bar{R}(t)\right| \ll 1\right)$, the normal-stress condition can be expanded as a Taylor series in $b_{n}(t) / \bar{R}(t)$. At leading order, this procedure yields an evolution equation for $\bar{R}$ that has the same form as equation (1). At $O\left(b_{n}(t) / \bar{R}\right)$, this yields an ordinary differential equation (ODE) for $b_{n}(t), \ddot{b_{n}}+(2 \dot{\bar{R}} / \bar{R}) \dot{b}_{n}+\left[(1-n)(\overline{\bar{R}} / \bar{R})+n\left(n^{2}-1\right)\left(\gamma /\left(\rho \bar{R}^{3}\right)\right)\right] b_{n}=0$.

An initial asymmetry excites vibrations whose amplitudes freeze (equation (3) in main text) and whose frequencies chirp (equation (4)) as $\bar{R} \rightarrow 0$. The coefficien of the $b_{n}$ term in the ODE controlling $b_{n}(t)$ has two contributions. The first term is due to the acceleration of the interface on average and is proportional to $\bar{R} / \bar{R}$. The second term is due to the stabilizing effect of line tension and is proportional to $\gamma /\left(\rho \bar{R}^{3}\right)$. As $\bar{R}$ goes to zero, both terms diverge but the second term diverges much more slowly than the first term. As a result, the effect of surface tension on the vibration becomes negligible near the singularity. To see this, first note that $\bar{R}$ evolves as $\rho \pi \bar{R}^{2} \bar{R}^{2} \ln \left(R_{\infty} / \bar{R}\right)=E$ (equation (2)) as $\bar{R}$ goes to zero. As a logarithmic variation changes very slowly, a good estimate for the leading-order behaviour of $\bar{R}$ is simply $\bar{R} \approx \sqrt{A\left(t_{*}-t\right)}$ with $A=\sqrt{4 E /\left(\rho \pi \ln \left(R_{\infty} / \bar{R}\right)\right)}$. With this leading-order behaviour, we then see that the $\ddot{\bar{R}} / \bar{R}$ term diverges approximately as $1 /\left(t_{*}-t\right)^{2}$, more rapidly than the $O\left(1 /\left(t_{*}-t\right)^{3 / 2}\right)$ divergence of the $\gamma /\left(\rho \bar{R}^{3}\right)$ term. In other words, as $\bar{R} \rightarrow 0$ the ODE for $b_{n}(t)$ simplifies to $\ddot{b_{n}}-\dot{b}_{n} /\left(t_{*}-t\right)+\left[(n-1) /\left(4\left(t_{*}-t\right)^{2}\right)\right] b_{n}=0$, an equi-dimensional, second-order ODE supporting solutions in the form of $\left(t_{*}-t\right)^{m}$ Solving the simplified ODE yields $m= \pm i \sqrt{n-1} / 2$, corresponding to vibrations whose amplitudes do not change over time. Eliminating $t_{*}-t$ and working with $b_{n}(\bar{R}(t))$ instead yields $\mathrm{d} a_{n} / \mathrm{d} t \rightarrow 0$ as $\bar{R}$ goes to zero, equivalent to equation (3). We also find that the $n$th vibrational mode chirps $\mathrm{d} \phi_{n} / \mathrm{d} t=\sqrt{n-1}(\overline{\dot{R}} / \bar{R})$ as $\bar{R}$ goes to zero, corresponding to a logarithmic variation in the phase variable $\left(\phi_{n}(t)=\sqrt{n-1} \ln [\bar{R}(t) / \bar{R}(t=0)]+\phi_{n}(t=0)\right)$.

Theory curve in Fig. 1 c. To generate the theory curve, we first chose a value for $E$ that enables equation (2), when starting with the initial value of the minimum neck radius, that is, $R(t=240 \mu \mathrm{s})$, to reproduce the observed closure time $t_{*}$. We then generate $R(z, t=0)$ by fitting a smooth curve through the experimental profile at $240 \mu$ s (cubic spline). Finally, using $R(z, t=0)$ as our initial condition, we solve equation (2) at different heights and plot the outcome at successive times.

Theory curve in Fig. 4. The linear stability curve is obtained by solving the unsimplified ODE for $b_{n}(t)$. The values of the coefficients in the ODE, which depend on $\bar{R}, \gamma$ and $n$, are fixed as follows. The mode number $n=2$. The average radius $\bar{R}$ is governed by equation (2), with $E$ again chosen to reproduce the observed closure time $t_{*}$ for the slot-nozzle experiment. To relate the value of $\gamma$, the 2D line tension, to $\sigma$, the surface tension at the air-water interface, we note that, in the experiment, the longitudinal radius of curvature, corresponding to the radius of a circle fitted to the neck minimum along the $r-z$ plane, is linearly proportional to the average neck radius at the minimum. Thus the Laplace pressure contribution for the three-dimensional neck shape at the minimum has the form $\sigma(\kappa-c / \bar{R}(t))$. From the data, the proportionality constant $c$ is approximately 0.36 . Requiring that the $2 \mathrm{D}$ line tension $\gamma$ assumes a value that gives the correct Laplace pressure contribution at leading order in the normal-stress balance, that is, $\gamma / \bar{R}(t)=\sigma(1-c) / \bar{R}(t)$, yields $\gamma=46$ dynes $\mathrm{cm}^{-1}$. Finally, as the equation is second order in time, a unique time evolution requires two initial conditions, corresponding to fixing values of $a_{2}$ and $\phi_{2}$ at a specific value of $\bar{R}$. Here we fix $a_{2}$ and $\phi_{2}$ so that the theory curve coincides with the measurements at the maximum of $\Delta R(\bar{R})$, roughly where $\bar{R}$ is $100 \mu \mathrm{m}$.

Received 22 July 2009; accepted 19 February 2009; published online 6 April 2009

\section{References}

1. Drazin, P. G. \& Johnson, R. S. Solitons: An Introduction (Cambridge Univ. Press, 1989).

2. Barenblatt, G. I. Scaling, Self-Similarity and Intermediate Asymptotics (Cambridge Univ. Press, 1996).

3. Shi, X. D., Brenner, M. P. \& Nagel, S. R. A cascade of structure in a drop falling from a faucet. Science 265, 219-222 (1994).

4. Cohen, I., Brenner, M. P., Eggers, J. \& Nagel, S. R. Two fluid snap-off problem: Experiments and theory. Phys. Rev. Lett. 83, 1147-1150 (1999).

5. Zhang, W. W. \& Lister, J. R. Similarity solutions for capillary pinch-off in fluids of differing viscosity. Phys. Rev. Lett. 83, 1151-1154 (1999).

6. Longuet-Higgins, M. S., Kerman, B. R. \& Lunde, K. The release of air bubbles from an underwater nozzle. J. Fluid Mech. 230, 365-390 (1991).

7. Oguz, H. N. \& Prosperetti, A. Dynamics of bubble growth and detachment from a needle. J. Fluid Mech. 257, 111-145 (1993).

8. Plesset, M. S. \& Prosperetti, A. Bubble dynamics and cavitation. Ann. Rev. Fluid Mech. 9, 145-185 (1977).

9. Strutt, J. W. The Theory of Sound (Macmillan, 1877).

10. Burton, J. C., Waldrep, R. \& Taborek, P. Scaling and instabilities in bubble pinch-off. Phys. Rev. Lett. 94, 184502 (2005).

11. Gordillo, J. M., Sevilla, A., Rodríguez-Rodríguez, J. \& Martínez-Bazán, C. Axisymmetric bubble pinch-off at high Reynolds numbers. Phys. Rev. Lett. 95, 194501 (2005).

12. Keim, N. C., Moller, P., Zhang, W. W. \& Nagel, S. R. Breakup of air bubbles in water: Memory and breakdown of cylindrical symmetry. Phys. Rev. Lett. 97, 145503 (2006).

13. Bergmann, R. et al. Giant bubble pinch-off. Phys. Rev. Lett. 96, 154505 (2006).

14. Thoroddsen, S. T., Etoh, T. G. \& Takehara, K. Experiments on bubble pinch-off. Phys. Fluids 19, 042101 (2007).

15. Eggers, J., Fontelos, M. A., Leppinen, D. \& Snoeijer, J. H. Theory of the collapsing axisymmetric cavity. Phys. Rev. Lett. 98, 094502 (2007).

16. Lewis, D., Marsden, J., Montgomery, R. \& Ratiu, T. The hamiltonian structure for dynamic free boundary problems. Physica D 18, 391-404 (1986).

17. Ott, E. Chaos in Dynamical Systems (Cambridge Univ. Press, 2002).

18. Whitham, G. B. A new approach to problems of shock dynamics. Part 1. Two-dimensional problems. J. Fluid Mech. 2, 145-171 (1957).

19. Evans, A. K. Instability of converging shock waves and sonoluminescence. Phys. Rev. E 54, 5004-5011 (1996).

20. Plewa, T., Calder, A. C. \& Lamb, D. G. Type la supernova explosion: Gravitationally confined detonation. Astrophys. J. 612, L37-L40 (2004).

21. Kadanoff, L. P. Computational scenarios. Phys. Today 57, 10-11 (2004).

22. Maeda, K. et al. Asphericity in supernova explosions from late-time spectroscopy. Science 319, 1220-1223 (2008).

23. Lohse, D. et al. Impact on soft sand: Void collapse and jet formation. Phys. Rev. Lett. 93, 198003 (2004).

24. Royer, J. R. et al. Formation of granular jets observed by high-speed x-ray radiography. Nature Phys. 1, 164-167 (2005).

25. Rygg, J. R. et al. Proton radiography of inertial fusion implosions. Science 319, 1223-1225 (2008).

\section{Acknowledgements}

We thank K. Turitsyn, L. Lai and S. Hilgenfeldt for comments. This work was supported by NSF-MRSEC No. DMR-0820054, the Keck Initiative for ultrafast imaging (University of Chicago) and a US Department of Education teaching fellowship (L.E.S.).

\section{Additional information}

Reprints and permissions information is available online at http://npg.nature.com/ reprintsandpermissions. Correspondence and requests for materials should be addressed to W.W.Z. 